\title{
Le critique est-il metteur en scène? Relire la querelle d'Horace avec les philosophies de la fiction
}

\section{Aurélien Maignant}

\section{OpenEdition}

1 Journals

Édition électronique

URL : https://journals.openedition.org/edl/3221

DOI : $10.4000 /$ edl.3221

ISSN : 2296-5084

Éditeur

Université de Lausanne

\section{Édition imprimée}

Date de publication : 24 septembre 2020

Pagination : 229-248

ISBN : 978-2-940331-74-1

ISSN : 0014-2026

Référence électronique

Aurélien Maignant, « Le critique est-il metteur en scène? Relire la querelle d'Horace avec les philosophies de la fiction », Etudes de lettres [En ligne], 313 | 2020, mis en ligne le 24 septembre 2021, consulté le 25 septembre 2021. URL : http://journals.openedition.org/edl/3221 ; DOI : https://doi.org/ 10.4000/edl.3221

\section{(C) Études de lettres}




\section{LE CRITIQUE EST-IL METTEUR EN SCÈNE? RELIRE LA QUERELLE D'HORACE AVEC LES PHILOSOPHIES DE LA FICTION}

Que faisons-nous aux fictions dramatiques lorsque nous parlons d'elles? Inspiré des travaux sur la critique interventionniste (Bayard, Saint-Gelais) et des philosophies de la vérité dans la fiction (Lewis, Walton), la présente contribution étudie comment le commentaire d'une pièce engage une représentation mentale de la scène qui pourrait s'apparenter à un acte créatif. Pour illustrer le propos, seront passées en revue différentes interprétations avérées depuis la fin du XIX ${ }^{\mathrm{e}}$ siècle d'un texte classique, le Horace de Corneille, en traquant dans leur discours interprétatif les symptômes de mises en scènes possibles. L'enjeu est d'avancer quelques pistes pour une réflexion sur la porosité entre interprétation et production de fiction.

\section{Le hiatus d'Horace}

Peut-on parler d'une pièce sans devenir metteur ou metteuse en scène? C'est la question qui nous intéressera ici, plus pour l'itinéraire qu'elle invite à emprunter qu'avec la prétention d'y apporter une réponse. Interroger l'effet du commentaire sur la fiction revient à questionner les fondements de l'herméneutique; que faisons-nous aux pièces lorsque nous parlons d'elles? Si le commentaire constitue le principe actif du rapport d'une société à ses œuvres, c'est que la fiction, même théâtrale, est d'abord une expérience solitaire. Ce que nous pouvons pluraliser dans l'interdiscours social, c'est la manière dont nous racontons cette expérience. Cette contribution englobera sous l'appellation de "critique» l'ensemble des lecteurs/lectrices ou des spectateurs/spectatrices 
produisant des énoncés sur une fiction dramatique, et se demandera en quoi leur activité peut être rapprochée de celle des metteurs en scène. On prendra un exemple classique, l'Horace de Corneille (1640) ${ }^{1}$, et en parcourant plusieurs commentaires historiquement avérés depuis la fin du $\mathrm{XIX}^{\mathrm{e}}$ siècle, on cherchera à en produire une description qu'on dira métaherméneutique, un terme proposé en 2014 par la narratologue Liesbeth Korthals Altes et défini comme un enjeu important pour l'avenir des théories et des philosophies du récit et de la fiction; dans son optique, la recherche doit en effet envisager une étude attentive de l'interprétation des œuvres dans ce qu'elle a de dynamique, sans aucune hiérarchisation, et en cherchant à mettre en lumière les paramètres conditionnant la production du sens des objets culturels ${ }^{2}$.

Si l'exemple est propice à une telle étude, c'est que depuis sa création, Horace a donné lieu à de nombreux débats interprétatifs. Pour la présenter rapidement, la pièce est une tragédie sur fond de guerre entre Rome et Albe, dont l'action repose sur un carré amoureux déchiré par le devoir patriotique: Horace et Camille, un frère et une sœur romains, respectivement époux et promise d'une sœur et d'un frère albains, Sabine et Curiace. Pour éviter que les différends entre les deux cités n'engendrent un massacre, celles-ci élisent deux champions qui décideront de l'avenir du conflit dans un duel à mort. Ceux qui ne connaissent pas la pièce l'auront sans doute deviné, les élus sont Horace et Curiace. Horace rentre victorieux du combat après avoir tué Curiace, et doit affronter la réaction de sa sœur Camille. Un événement curieux se produit alors. Face à une douleur pourtant compréhensible, Horace déclare assez brusquement: «à la patience ma raison fait place» et assassine sa sœur d'un coup d'épée ${ }^{3}$. Étrange sentence; en quoi abattre Camille de sang-froid serait-il un acte de "raison"? L'acte suivant, le cinquième et dernier, est occupé par le procès du meurtrier qui, après divers plaidoyers et réquisitoires, sera finalement acquitté par le Prince de Rome en vertu du service

I. P. Corneille, Horace, éd. par M. Escola.

2. L. Korthals Altes, Ethos and Narrative Interpretation. Sa perspective est très proche de ce que R. Baroni appelle une "critique polyphonique», en défendant notamment, comme on le propose ici, la nécessité d'une approche métaherméneutique des critiques et des commentateurs académiques, comme sources de réceptions manifestant des positions dans l'espace des interprétations possibles (cf. Baroni, «La guerre des voix»).

3. P. Corneille, Horace, acte IV, p. 122. 
qu'il a rendu à la patrie en triomphant de Curiace. Ce meurtre constitue un hiatus important qui n'a cessé d'interroger la critique. On observera par la suite comment la pluralité d'interprétations de la pièce pourrait partiellement être étudiée comme une pluralité de «remises en scène» permettant de justifier un acte que le texte n'explique pas. Mais avant de livrer cette étude, on aura besoin d'un rapide détour sur la notion d'interprétation dans le cas des textes dramatiques.

\section{De l'inférence à l'ingérence}

On pourrait aborder l'idée d'«interprétation» d'un texte dramatique en quatre acceptions polarisées:

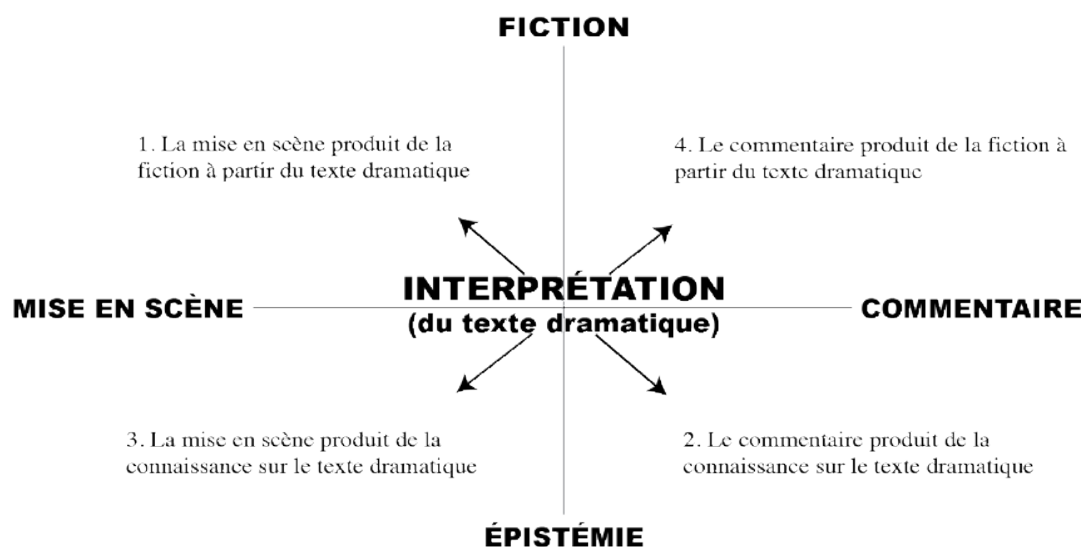

On dira a priori (position 1) qu'un metteur en scène livre «son interprétation" d'Horace, ce qui désigne une démarche plus spécifiquement qualifiée d'«adaptation». Dans cette acception, l'interprète n'est pas tant celui qui produit du savoir sur l'œuvre, que celui qui en produit de la fiction (un nouveau spectacle d'Horace). À l'autre pôle (position 2), lorsqu'un chercheur écrit un livre ou un article sur Horace, on dira qu'il en donne "son interprétation». Dans ce contexte, l'interprète est une autorité dont la fonction n'est pas de produire de la fiction, mais bien de la connaissance sur le texte. Mais dépassons prestement l'évidence; cette polarité entre épistémie et fiction est de plus en plus contestée dans les approches contemporaines de la fiction. 
Dans une réflexion sur la théorie du théâtre, Romain Bionda ${ }^{4}$ remet en question l'idée même que nous puissions trouver la "vérité» d'une situation fictionnelle dans un texte dramatique. On sait l'importance de la vérité dans la fiction ${ }^{5}$ pour les commentateurs, cette catégorie d'interprètes qu'on ne cesse de comprendre comme des enquêteurs ${ }^{6}$. Lorsque Bionda conclut sa réflexion en soulignant que chaque nouvelle mise en scène d'un texte pourrait être appréhendée comme preuve d'une lecture possible, il esquisse un rapprochement entre l'activité des metteurs en scène et celle des commentateurs également engagés dans une enquête, à l'issue de laquelle ils opéreront une reconfiguration de l'œuvre pour en produire du sens. Sous cet angle, on pourrait tout à fait assimiler une mise en scène d'Horace renversant l'autorité du frère sur la sœur, et un commentaire académique de la pièce issu par exemple des gender studies. Dans ce cas, le metteur en scène produirait de la connaissance en construisant sa fiction scénique (position 3).

Le renversement inverse de la polarité nous occupera principalement; peut-on envisager que le commentateur (académique par exemple) produise de la fiction en construisant du savoir sur l'œuvre (position 4)? Cette hypothèse est très sérieusement avancée par Richard Saint-Gelais dans un ouvrage (Fictions transfuges) consacré, pour le dire trop rapidement, aux limites de la fiction et à la manière dont elles peuvent être transgressées. La dernière partie, "Critique et transfictionnalité", épingle une pratique fréquente chez les critiques, à savoir que leur discours sur les œuvres modifie le monde fictionnel originel, car il est souvent contraint d'y ajouter des éléments:

[...] une partie de la diégèse ${ }^{7}$ dépend de l'intervention du lecteur, qui développe silencieusement un discours hétérogène et continu [...] ce

4. R. Bionda, «La vérité du drame».

5. Il est l'un des premiers à appliquer au texte dramatique une problématique connues des théories de la fiction, souvent réticentes à aborder des corpus théâtraux. Cf. notamment Lewis, "Truth in Fiction", ou Walton, Mimesis as Make-Believe, on the Foundation of Representational Arts.

6. Cf. les célèbres réflexions d'Eco (Lector in fabula) et la forme qu'elles prennent dans les travaux de Bayard (Qui a tué Roger Ackroyd?) ou d'Escola ("Pierre Bayard contre Hercule Poirot, derniers rebondissements dans l'affaire Ackroyd »; "Changer le monde»).

7. Dans la narratologie classique, la diégèse désigne l'espace-temps signifié par les signifiants du récit. Sans entrer dans les détails d'un vif débat et à la suite des théories 
discours je propose de le penser à l'aide de la notion de parafictionnalisation, de manière à souligner que le lecteur ne le produit que pour le verser aussitôt au compte de la fiction. Se dire, par exemple, que Holmes est plus futé que Watson, que Hamlet est névrosé ou que c'est l'amour de Sonia qui décide Raskolnikov à confesser son crime, c'est contribuer chaque fois à la diégèse par des propositions ${ }^{8}$.

Cette approche de la parafictionnalisation entérine un lien indéfectible entre production de sens et production de fiction, justifié par la nature co-construite du monde fictionnel. En raison de cette justification, la proposition de Saint-Gelais apparaît comme la formulation narratologique d'un constat sur la nature des mondes déjà éprouvé par les philosophies analytiques de la fiction. Rappelons qu'il existe un débat entre partisans de la complétude ou de l'incomplétude des mondes fictionnels. Les seconds, comme Lubomir Dolelzel ${ }^{9}$, adoptent une attitude logicienne à l'égard des mondes fictionnels et les décrivent comme des ensembles incomplets. Pour Dolezel, si le texte ne spécifie pas le nombre d'enfants de Lady Macbeth, c'est qu'une telle proposition a une vériconditionnalité nulle; elle n'a pas de vérité dans la fiction. Les premiers rétorquent aux seconds que leur argument perd sa pertinence en dehors la logique propositionnelle. Selon eux, l'incomplétude des mondes va à l'encontre de l'expérience du lecteur et du spectateur qui, par un ensemble de processus cognitifs, accordent aux mondes fictifs (et aux personnages qui les habitent) une ontologie propre, une «thèse d'existence intuitive». Dans les termes de Marie-Laure Ryan:

Si elle semble logiquement valable, la thèse de l'incomplétude des mondes fictionnels échoue sur le plan phénoménologique, car elle ne rend pas compte de l'expérience du lecteur. Nous n'imaginons pas que les héroïnes de Mme de La Fayette manquent de visage [...]. Dans notre acte d'imagination, ces manques sont une question épistémologique et non ontologique ${ }^{10}$.

C'est la position que nous adopterons; la participation imaginative des récepteurs confère une vériconditionnalité aux énoncés laissés

de la fiction récentes, on parlera ici de «monde» ou d' «univers» fictif.

8. R. Saint-Gelais, Fictions transfuges, p. 457 sq.

9. L. Dolezel, Heterocosmica.

Io. M.-L. Ryan, «Cosmologie du récit», p. 57. 
indéterminés par le texte. Dans une perspective herméneutique, cette indétermination crée justement un espace phénoménologique pour l'enquête interprétative; le manque épistémique rend la parafictionnalisation nécessaire. Le concept même de "vérité dans la fiction" a d'abord été problématisé par David Lewis ${ }^{11}$, pour sortir le débat logicien sur les énoncés fictionnels de la vériconditionnalité référentielle; il n'était plus question d'étudier la validité des énoncés fictionnels par rapport au monde réel, mais relativement au monde de fiction dans lequel ils sont actuels ${ }^{12}$. Dans le développement ultérieur que lui donne Kondall Walton, la vérité dans la fiction devient le mode d'engagement imaginatif des récepteurs. Redéfinissant la mimésis ${ }^{13}$ comme un "faire croire», le philosophe fait de l'enquête sur la vérité dans la fiction une description possible de l'immer$\operatorname{sion}^{14}$. À ses yeux, le principe actif de la représentation est de constituer un ensemble d'artefacts comme déclencheurs (props) d'un état de croyance chez le spectateur. Tout l'enjeu est alors d'étudier en quoi ces croyances dans la fiction s'apparentent ou divergent de nos croyances sur des objets réels. Le champ de la réflexion s'est ensuite étendu chez les analytiques autour de la question des inférences possibles, soit des limites qu'il convient de poser à cette enquête. Confronté à un conflit entre ce que deux personnages croient vrai, par exemple les motivations du crime d'Horace, les récepteurs mobiliseraient leurs connaissances sur le réel pour donner du sens à la situation. Dans une idée similaire, Thomas Pavel ${ }^{15}$ considère que nos savoirs individuels sont le support de nos inférences fictionnelles; lorsque nous sommes confrontés à un problème dans la fiction, nous tentons de le résoudre au moyen de notre encyclopédie personnelle. La proposition rejoint celle de Ryan qui propose d'appeler "principe d'écart minimal» le réflexe imaginaire des lecteurs

II. D. Lewis, "Truth in Fiction».

I2. Démarche qui vise aussi à sortir le débat des approches logico-sémantiques de l'énoncé de fiction (fertiles au début du $\mathrm{XX}^{\mathrm{e}}$ siècle) selon lesquelles toute phrase fictionnelle, ne référant à aucun élément du monde réel, serait «mensongère» ou simplement "vide» (voir notamment B. Russell, «De la dénotation»). Envisager la vériconditionnalité des énoncés fictionnels dans leur propre monde empêche de poser le problème en termes d'imitation ou de copie de la réalité.

13. L'enjeu de la présente réflexion n'est pas de rendre compte des nombreux débats sur le concept; voir la redéfinition qu'en a proposée M. Groneberg in "La mimesis».

14. K. Walton, Mimesis as Make-Believe, on the Foundation of Representational Arts.

15. Th. Pavel, Univers de la fiction. 
qui supposent que le monde de fiction obéit aux mêmes lois que leur réalité jusqu'à l'éventuelle mention du contraire. Dans ces deux optiques, un lecteur d'Horace pourra par exemple en appeler à ses connaissances psychologiques sur le traumatisme pour justifier l'acte d'Horace. Pour sa part, Gregory Currie ${ }^{16}$ défend ce qu'on nomme généralement «le principe de croyances mutuelles", selon lequel la vérité d'un monde serait coextensive aux connaissances de l'auteur et à la construction de ses croyances par les lecteurs; il serait ainsi faux de diagnostiquer un complexe d'CEdipe à Horace, puisque la psychanalyse était inconnue de Corneille. Walton argumente en faveur d'un principe plus large - dit « de réalité" - selon lequel la vérité d'une interprétation serait dépendante de nos connaissances du monde réel, sans qu'il faille y intégrer nécessairement une problématisation historique; à ce titre, la vérité dans la fiction d'un possible complexe d'CEdipe d'Horace serait dépendante de la vérité de la psychanalyse dans le monde réel. Plus récemment, la narratologie cognitive commence à travailler une idée idoine en étudiant les mécanismes cognitifs de reconstruction du storyworld (selon la terminologie de David Herman ${ }^{17}$. Les récepteurs seraient en perpétuelle réorganisation d'indices sur différentes dimensions du monde (qui, quoi, quand, où, etc.) ; ils produiraient une image mentale basée sur des "scripts» (soit des structures narratives connues) ${ }^{18}$ et des «cadres» (des modes de représentation de l'espace-temps) ${ }^{19}$. Dans le cas d'Horace, on dira par exemple qu'un récepteur peut comprendre le geste criminel en identifiant le scénario culturel de la douleur lyrique ou du fratricide symbolique, tout en ayant suffisamment d'indices pour reconstruire un cadre historico-politique expliquant l'acquittement d'Horace pour service rendu à l'État.

Toutes ces approches ont en commun d'associer de manière inextricable l'enquête (production de connaissance sur l'œuvre) et les processus de simulation mentale du monde de fiction. On peut ainsi retrouver le principe de la parafictionnalisation, tel que le théorise Saint-Gelais. Dans une acception radicale, on dira que la logique même du commentaire de fiction induit une modification du monde fictif, l'inférence reposerait

I6. G. Currie, The Nature of Fiction.

17. D. Herman, Storytelling and the Sciences of Mind.

I8. Cf. notamment Baroni, Les rouages de l'intrigue, p. 75-80.

19. M. Jahn, "Frames, Preferences, and the Reading of Third-Person Narratives». 
sur une ingérence. Ce principe, nous nous proposons de l'appliquer au cas spécifique de la fiction théâtrale, trop rarement abordé dans la philosophie de la fiction.

Le texte de théâtre a en effet la particularité de faire toujours signe vers une scène possible, sans que le rapport de l'un sur le monde de l'autre ne soit jamais rigide (un des constats à la base de la perspective de Bionda). On voudra donc repenser la parafictionnalisation des commentateurs de théâtre qui, enquêtant sur un monde dont la vérité textuelle est labile, devraient produire une fiction mentale pour mener à bien leur enquête épistémique. On montrera que dans certains cas, la reformulation du contenu fictif pourrait donner lieu à une mise en scène possible du texte - soit que la trace de l'activité de simulation présente des similitudes avec la construction d'une fiction scénique. De cette nécessité, philosophiquement justifiée par l'incomplétude des mondes fictifs, la réception conflictuelle d'Horace constitue un exemple fertile. Précisons que la réflexion vaut pour le principe même de l'énoncé sur la fiction, celui des spectateurs comme celui des scholars. Si le corpus choisi est essentiellement constitué de textes académiques, c'est parce que les sources écrites sont plus aisément accessibles.

\section{Remettre Horace en scène}

Interpréter l'incompréhensible geste criminel d'Horace engage la vériconditionnalité des intentions du personnage, soit à tenter de comprendre qui est Horace. Les motivations du tueur et son caractère constituent l'un des principaux espaces de parafictionnalisation, ce qui montre bien que la pièce n'y apporte aucune réponse satisfaisante. Pour autant, presque tous les personnages informent le lecteur, à un moment où à un autre, de leur représentation d'Horace. Sans surprise, ces représentations divergent largement, ce que Marc Escola analyse de manière générale dans la tragédie française du XVII e comme une friction entre univers de croyances divergents:

Peut-être même faut-il décrire le conflit dramatique comme friction entre univers de croyances divergents, et le tragique comme divorce entre mondes irréconciliables (par quoi l'on se vouerait sans doute à 
retrouver par des voies grammaticales la conception hégélienne du tragique) ${ }^{20}$.

Sans conteste, le tragique horacien repose lui aussi sur une friction de ce type. Il suffit de comparer les univers de croyances sur Horace de Valère (un noble romain amoureux de Camille) et de Sabine (son épouse et sœur de Curiace) :

SABINE: Quel ennemi choisir d'un Époux ou d'un Frère?

La Nature ou l'Amour parle pour chacun d'eux,

Et la loi du devoir m'attache à tous les deux,

Sur leurs hauts sentiments, réglons plutôt les nôtres,

Soyons femme de l'un ensemble et sœur des autres,

Regardons leur honneur, comme un souverain bien,

Imitons leur constance et ne craignons plus rien.

[...] Et sans considérer aux dépens de quel sang,

Leur vertu les élève en cet illustre rang (vv. 716-730).

VALÈRE: Quel sang épargnera ce barbare vainqueur,

Qui ne pardonne pas à celui de sa sœur

Et ne peut excuser cette douleur pressante

Que la mort d'un amant jette au cœur d'une amante, [...]

Combien un pareil coup est indigne d'un homme;

$[\ldots]$

Vous verriez un beau sang, pour accuser sa rage,

D'un frère si cruel rejaillir au visage;

Vous verriez des horreurs qu'on ne peut concevoir (vv. 1715-1740).

Deux univers de croyances, deux portraits d'Horace que tout oppose. Pour Sabine, Horace est vertueux, pour Valère, il n'est qu'un monstre, à tel point qu'on pourra trouver une symétrie étonnante dans ces descriptions. La «constance» que lui attribue son épouse contraste avec la «rage» que Valère lui attribue, la première trouve des «hauts sentiments" à un homme que le second juge "barbare», et là où elle voit de la "vertu», il n'observe que des «horreurs». Ce type de conflit entre univers de croyances fait partie des dispositifs narratifs qui contraignent les critiques à la parafictionnalisation; pour interpréter l'acte d'Horace, il faut définir le personnage à l'intérieur de cette friction représentationnelle.

20. M. Escola, «Le sixième acte», p. 152. 
L'enjeu du commentaire sera donc de stabiliser une version compréhensible de ce récit dont la vérité est labile, structurée par un ensemble de perspectives subjectives contradictoires.

On examinera six des versions stabilisées, telles qu'elles apparaissent dans la réception d'Horace de la fin du XIX ${ }^{\mathrm{e}}$ siècle jusqu'à la deuxième moitié du $\mathrm{XX}^{\mathrm{e} 21}$. On s'attardera particulièrement sur le sens qu'elles donnent à la scène du meurtre, qu'elles la commentent explicitement ou l'illustrent par leurs portraits d'Horace et Camille. Précisons bien que toutes commentent le texte de Corneille, et non un spectacle en particulier. La parenté de leur activité avec celle des metteurs en scène sera soulignée par une question simple: leur représentation mentale de la fiction pourrait-elle se traduire en consignes de jeu?

Un premier commentaire sera celui de Francisque Sarcey, présenté dans un ouvrage sobrement intitulé La tragédie publié en 1887. Dans un extrait mis en lumière par Escola ${ }^{22}$, le critique se livre à une catégorie de parafictionnalisation que Saint-Gelais nomme «contrefictionnalisation». Le terme désigne, pour le dire vite, le détour que peut faire le commentaire par une version alternative de l'histoire, souvent mobilisée pour résoudre un problème. À la différence de l'hypothèse esquissée plus tôt, cette version n'est pas celle que le critique établit, mais une autre qu'il sait pertinemment fausse, construite uniquement pour illustrer la version qu'il considère comme vraie:

Supposez que Corneille nous eût fait de son Horace une âme très chevaleresque, de sentiments nobles et fiers, patriote ardent, soldat valeureux, mais contenu et superbe [...] cela était possible, j'ajouterai même, cela était vraisemblable. [...] Oui, mais si c'est là le caractère d'Horace dans la pièce, que devient le dénouement? Le dénouement exigeait absolument qu'Horace fut non seulement un vaillant homme de guerre [...] mais également un patriote enragé [...] avec une impétuosité aveugle; plein de jactance et de sottise, sans ménagement pour personne ${ }^{23}$.

2I. Je m’appuie ici sur le dossier de réception constitué par Marc Escola dans son édition du Horace de Corneille (p. 181-191), qui problématise ce débat critique dans sa propre perspective.

22. P. Corneille, Horace, p. 182.

23. F. Sarcey, La tragédie, p. 19 sq. 
Accordons-nous d'abord avec Sarcey sur ce constat toujours prégnant: $\mathrm{du}$ "caractère» d'Horace dépend le sens qu'il faut donner à la pièce. Dans sa contrefictionnalisation, le critique envisage explicitement une version du monde fictif proche de celle de Sabine, où Horace est honorable, et il cherche à montrer que la vérité d'une telle affirmation n'aurait pas mené au dénouement tel qu'on le connait. On ne peut toutefois nier que la version qu'il croit vraie repose elle aussi sur des parafictionnalisations concernant le caractère du meurtrier. Le critique esquisse une interprétation faisant d'Horace un patriote cruel, froid et sans intelligence, que rien n'excuse et pour qui le public doit ressentir de la répulsion. Inévitablement, son opération de lecture fait exister un plateau de théâtre hypothétique sur lequel le personnage serait donné à voir dans sa colère. Sa description de la réaction d'Horace avoisine tout à fait la consigne de jeu lorsqu'il voit en lui une "impétuosité aveugle», consigne de jeu qui trouve son pendant quelques lignes auparavant quand le critique décrit la réaction de "sa» Camille comme «l'action la plus naturelle qui soit ${ }^{24}$.

La version mise en scène (si l'on me pardonne la métaphore) de Sarcey pourrait être comparée à celle que produit un autre commentaire, proposé par Robert Brasillach dans Corneille (1938). Acquis dès cette date à l'idéologie nazie qui lui vaudra d'être fusillé à la Libération, Brasillach interprète le geste d'Horace au prisme du contexte politique contemporain. L'idée maitresse de son commentaire est de faire de la pièce une allégorie des guerres idéologiques modernes, dans laquelle Albe représenterait la faiblesse des régimes démocratiques face aux pouvoirs totalitaires (représentés par Rome, métonymiquement par Horace). On retrouve dans sa version une description du caractère d'Horace qui produit certains échos avec celle de Sarcey. Pour Brasillach, Horace incarne le "patriotisme ardent du jeune nazi ${ }^{25}$, interprétation qui esquisse un jeu des plus féroces où Sarcey retrouverait sans doute l'impétuosité qu'il voit lui aussi en Horace. Brasillach commente la réaction de Camille à l'aune de son portrait global des albains en faibles soldats démocrates: résignée, sans volonté propre. La Camille de Brasillach diffère ici de celle de Sarcey; il n'est plus question d'une émotion naturelle, plutôt d'un abattement innocent et frêle.

24. Ibid., p. 19.

25. R. Brasillach, Corneille, p. 139. 
Ces deux Horaces impétueux contrastent fortement avec un troisième, proposé par Gustave Lanson dans un ouvrage également intitulé Corneille (1898). Il y interprète l'ensemble de la pièce à l'aune de la vision cornélienne de la rationalité: Horace et Camille sont deux fanatiques de la vertu dont les réactions sont parfaitement maîtrisées et rationnelles. Avec une précision certaine, il envisage une version de l'acte meurtrier où s'esquissent un troisième Horace et une troisième Camille. Nulle impétuosité dans son Horace qui «[...] tue [Camille] par raison; il n’y a pas là une folie féroce mais une froide justice ${ }^{26}$. On pourra reconnaître que la consigne de jeu n'est pas absolument claire, mais on admettra sans doute que le «jeu possible» est une modalité de divergence nette entre le Horace de Lanson et celui des deux commentateurs précédents. La situation est plus claire encore si l'on s'attarde sur l'interprétation que donne Lanson de l'acte de Camille qui, dans la tirade précédant le coup mortel, maudit sa Rome natale:

[...] sa malédiction sur Rome n'éclate point comme l'explosion involontaire d'une âme trop pleine; c'est une démarche calculée, à laquelle elle s'est mûrement excitée. Ce n'est point une folle douleur, mais une vendetta froide ${ }^{27}$.

Pas de "folle douleur", mais "une démarche calculée», "une vendetta froide"; tout concourt à produire une fiction scénique possible bien différente des deux précédentes. Comme chez les autres commentateurs, l'espace de la transaction parafictionnelle est aussi bien le caractère d'Horace que celui de Camille. À nouveau, on voit que le critique doit produire une représentation mentale des actions, et que certains aspects de cette représentation pourraient bien devenir des consignes de jeu.

Plus proche de nous, une quatrième version de la fiction a été proposée par Jacques Maurens, professeur spécialiste de Corneille, dans La tragédie sans tragique (1966). Sans entrer dans le détail, la pièce porte à ses yeux la marque du renouveau néo-stoïcien en vogue au XVII ${ }^{e}$ siècle. Par son action, Horace offre en miroir au Prince l'occasion d'exercer une morale stoïque avec une noblesse qui échapperait au "peuple stupide» ${ }^{28}$. À nouveau, on trouve dans l'ouvrage une interprétation s'appuyant sur

26. G. Lanson, Corneille, p. 104.

27. Ibid.

28. J. Maurens, La tragédie sans tragique, p. 269. 
une représentation très ocularisée de l'acte dans la fiction. L'Horace de Maurens n'est pas la brute aveuglée par son impétuosité mise en scène par Sarcey, mais il s'en approche. Type achevé de la générosité, il ne cède à la fureur que lorsque la malédiction de Camille excède sa personne propre et atteint son honneur de patriote:

[...] tant que l'outrage s'adresse à lui seul il arrive à se contenir par un effort qui rappelle sa générosité. Il ne frappe qu’après la malédiction contre Rome [...] parce que sa vertu farouche fait de ce meurtre un devoir patriotique ${ }^{29}$.

C'est peut-être une interprétation à ranger quelque part entre celle de Lanson et celles de Sarcey ou Brasillach; Horace est vertueux, mais cette vertu se manifeste dans la fureur. Concentrons-nous sur la scène possible de Lanson; comment ne pas voir une décision de metteur en scène dans ce personnage qui essaie de se contenir, puis qui n'y parvient plus et explose? Ici encore, rien dans le texte n'autorise un tel ajout au monde. La Camille de Maurens est d'un type nouveau, elle est certes assujettie à ses émotions, contrairement à celle de Lanson, mais son attitude est toute différente de la «résignation» qu'ont pu lui attribuer les autres critiques. Peu loquace à son sujet, Maurens la décrit toutefois comme explosive et "provocatrice» ${ }^{30}$, engageant sa scène possible vers un jeu encore différent des trois précédemment cités.

L'exemple de consigne de jeu "critique» le plus indiscutable de la Querelle d'Horace est peut-être à trouver dans un cinquième commentaire, proposé cette fois par Louis Herland, quelques années avant Maurens, dans un ouvrage intitulé Horace ou la naissance de l'homme (1952). Dans son interprétation, qui pour Escola hésite à se réclamer de la psychanalyse ${ }^{31}$, le crime d'Horace traduit le refoulement de la douleur d'avoir tué Curiace, et lui permet avant tout de ne plus douter de la légitimité de ce premier meurtre. Sa Camille est proche de celle de Sarcey quoique plus radicale, elle réagit de manière incontrôlable (Sarcey disait de la manière «la plus naturelle qui soit»), poussée par une "douleur

29. Ibid., p. 268.

30. Ibid., p. 267.

31. P. Corneille, Horace, p. 189, 
démente ${ }^{32}$. Plus intéressant, sa longue description du meurtre esquisse nettement une scène possible:

Que dis-tu malheureuse? [v. 1267] ce cri rauque inoubliable [...] où la douleur éclate à l'état pur. Corneille a voulu pousser au maximum la souffrance de son héros ${ }^{33}$.

La réplique d'Horace est immédiatement accompagnée d'une consigne de jeu ("ce cri rauque») portant sur le registre et le timbre vocal. Parafictionnalisation, participation mentale ou scène possible; son interprétation du sens du texte théâtral recourt indubitablement à la production d'une version ocularisable de la fiction scéniquement encodée.

Sixième et dernier exemple qui sera examiné ici, l'interprétation d'Horace proposée par Serge Doubrovsky dans Corneille et la dialectique du héros (1963). Pour lui, la pièce est une allégorie de la philosophie hégélienne, et le combat entre Horace et Camille doit être lu comme une manifestation de la "lutte à mort des consciences " ${ }^{34}$, théorisée comme mouvement dialectique par Hegel. La scène possible de Doubrovsky envisage un Horace éloigné lui aussi de toute barbarie, un frère tragique et généreux qui, en termes de jeu, s'illustre par son sens du sacrifice:

[...] ces appels du sentiment, il les a douloureusement, en lui-même, surmontés et exprimés [...] il demande à sa sœur de faire l'effort qu'il a fait sur lui-même ${ }^{35}$.

Le commentateur imagine alors une Camille en miroir, hérö̈que elle aussi, son héroïsme conditionnant le dépassement dialectique d'Horace:

[...] les valeurs du sentiment ne sont pas vécues par Camille que dans la honte mais en toute lucidité et la tête haute [...] elle cherche délibérément à se faire tuer ${ }^{36}$.

Si l'on avait le goût de l'excès, on pourrait presque assimiler l'expression courante "la tête haute» à une consigne de jeu. Avec plus de modération, on retiendra qu'une interprétation allégorique complexe et référencée,

32. L. Herland, Horace ou la naisssance de l'homme, p. 156.

33. Ibid.

34. S. Doubrovsky, Corneille et la dialectique du héros, p. 164.

35. Ibid., p. 163.

36. Ibid., p. 166. 
comme celle de Doubrovsky, ne peut faire l'économie d'une version mentale très concrète du monde de fiction, et par là l'évocation d'une scène possible: il faut que Camille «joue» avec dignité pour que l'interprétation hégélienne fonctionne. Ce constat, valable aussi pour les interprétations cartésienne ou néostoïcienne de Lanson et de Maurens, attire notre attention sur ce que signifie véritablement le lien entre production de connaissance sur l'œuvre et production de fiction; chaque commentaire construit sa propre version du monde et, dans le cas théâtral, elle fait parfois signe vers une mise en scène possible.

Pour conclure l'analyse, nous esquisserons une grille métaherméneutique reprenant, en les comparant, les différentes versions de la fiction construites depuis le XIX ${ }^{\mathrm{e}}$ siècle par les commentateurs d'Horace ${ }^{37}$. On nous pardonnera de simplifier chaque lecture pour produire une meilleure comparaison des transactions parafictionnelles. Classer ces six interprétations sur la seule dichotomie d'une réaction pulsionnelle ou rationnelle permet de les réduire à un degré minimal, où la divergence des versions est claire:

\begin{tabular}{||l||l||l|l||l|l||}
\hline Versions & Interprètes & $\begin{array}{l}\text { Réaction } \\
\text { de Camille }\end{array}$ & $\begin{array}{l}\text { Réaction } \\
\text { d'Horace }\end{array}$ & $\begin{array}{l}\text { Motivations } \\
\text { de Camille }\end{array}$ & $\begin{array}{l}\text { Motivations } \\
\text { d'Horace }\end{array}$ \\
\hline \hline V1 & $\begin{array}{l}\text { Brasillach } \\
(1938)\end{array}$ & pulsionelle & rationnelle & faiblesse & patriotisme \\
\hline \hline V2 & $\begin{array}{l}\text { Sarcey } \\
(1887)\end{array}$ & pulsionelle & pulsionelle & émotion & barbarie \\
\hline \hline V3 & $\begin{array}{l}\text { Lanson } \\
(1898)\end{array}$ & rationnelle & rationnelle & vengeance & devoir \\
\hline \hline V4 & $\begin{array}{l}\text { Herland } \\
(1952)\end{array}$ & pulsionelle & pulsionelle & amour & culpabilité \\
\hline \hline V5 & $\begin{array}{l}\text { Maurens } \\
(1966)\end{array}$ & pulsionelle & rationnelle & impuissance & vertu stoïque \\
\hline \hline V6 & $\begin{array}{l}\text { Doubrovs- } \\
\text { ky (1988) }\end{array}$ & rationnelle & rationnelle & $\begin{array}{l}\text { suicide } \\
\text { héroique }\end{array}$ & dépassement \\
héroïque
\end{tabular}

37. On propose ici une métaherméneutique déductive (modéliser la pluralité des interprétations avérées). Dans une précédente étude (Maignant, "Juger de la fiction»), nous avons proposé une approche inductive (modéliser la virtualité des lectures programmées par les formes). 
Il est indéniable que les versions de Herland et de Doubrovsky (V4 et 6) divergent au moins sur le degré de maîtrise de soi manifesté par Horace et Camille; elles sont sur ce point tout à fait incompatibles. En revanche, les mondes d'Horace décrits par Brasillach et Maurens (V1 et 5) sont compatibles par leurs descriptions de la réaction bien qu'incompatibles sur la question des motivations. La version de Lanson (V3), lorsqu'elle décrit deux personnages mus par leur seule raison, est proche de celle de Doubrosky (V6), et nous apprend que les lectures cartésienne et hégélienne construisent un monde fictif relativement commun (mais est-ce bien une surprise?). V3 (cartésianisme) n'ayant qu'un seul niveau de compossibilité avec V1 et V5, nous observons également que les lectures fasciste et néostoïcienne ne sont compatibles avec celle de Maurens qu'en intégrant dans le monde représenté la nécessité d'un personnage nonrationnel. Enfin, V3 et V6 étant tout à fait incompossibles avec V2 et V4, il semble que les lectures psychologiques et psychanalytiques engagent des représentations de l'univers fictionnel tout à fait divergentes des deux interprétations se revendiquant d'un modèle philosophique précis.

\section{Re-raconter le théatre}

Au final, s'il y a de la mise en scène dans la critique, ce n'est pas en raison d'une distinction institutionnelle ou praticienne, mais en partie à cause de ce que les philosophies de la fiction nomment «la complétude des mondes fictifs». On invitera ici à un dépassement du concept de parafictionnalisation proposé par Saint-Gelais - qui ne prétend d'ailleurs fonctionner que sur la base du donné sémantique textuel. Du point de vue cognitif, le terme en lui-même sous-entend une distinction trop nette entre ce qu'est la fiction et ce qui est autour (para). La réalité phénoménologique de l'interprétation semble bien plus complexe. Une approche métaherméneutique de la querelle d'Horace montre bien l'impossibilité d'une définition stable de ce qu'est le monde fictif de la pièce, plus précisément ici du caractère du personnage. Ceci s'explique par la nécessité dans laquelle se trouve l'interprétation d'établir une "version vraie» de la fiction, immédiatement empêchée par ce que cette vérité peut avoir de polyphonique ou de matriciel.

Aussi, de manière radicale, on pourrait envisager que la parafictionnalisation est intrinsèque au discours sur la fiction, étant donné la nécessité 
pour les commentateurs de reconstruire une version: leurs "vérités dans la fiction ». Sans aller jusqu' à cet extrême constructiviste ${ }^{38}$, il semble plus juste de mettre en lumière l'importance de la reconstruction de la fiction dans le discours, et par extension d'inviter à penser davantage la manière dont la production de connaissance est sous-tendue par celle d'une représentation mentale des situations mises en récit. Il est apparu ici que le cas du texte dramatique est encore plus prégnant. Si l'on admet, avec Denis Guénoun, que de la double narrativité du théâtre, le texte ne contient que le récit dramatique (auquel il manque le récit scénique), force est de constater qu'aucun des commentaires d'Horace n'a pu s'épargner la construction d'une scène possible (évidemment lacunaire), la reconstitution de quelques morceaux de cette narrativité scénique qui manque au texte. La présente réflexion s'axait sur la question du jeu, mais d'autres pistes restent à explorer pour étudier ces scénographies mentales, notamment l'espace dramatique, souvent implicitement reconstruit par les critiques avec, contre, ou autour de ce que dit le texte.

Soulignons pour finir que ces constats, outre le fait qu'ils invitent à un abandon de la distinction fréquente entre les lectures "ordinaires» et les lectures «savantes» (qu'on repense à Doubrovsky, dont l'allégorie hégélianiste reposait sur une question très «ordinaire" d'intention du personnage), ils engagent à repenser ce que le discours sur les œuvres peut avoir en lui-même de narratif. Ce pourrait être un premier axiome d'une herméneutique par le récit; et si cet impensable qu'est "le sens d'une œuvre» désignait en fait l'ensemble de ses re-narrations possibles? L'interprétation du texte dramatique se verrait ainsi obligée, le «remettant en scène", d'y rétablir une narrativité manquante. Voilà peut-être une réponse possible, issue des philosophies de la fiction, à la question qui occupait le début de cet article. Que faisons-nous aux pièces lorsque nous les interprétons? Nous les re-racontons, pour leur donner du sens ${ }^{39}$.

Aurélien Maignant

Université de Lausanne

38. Pour une défense de cette version radicale du constructivisme, cf. les approches sociologique de Fish (Quand lire c'est faire), psychanalytique de Bayard (Qui a tué Roger Ackroyd?), ou narratologique de Nünning ("Unreliable Compared to What?»).

39. Avec tous mes remerciements à Marc Escola pour sa relecture. 


\section{BIBLIOGRAPHIE}

\section{Textes}

Corneille, Pierre, Horace, éd. par Marc Escola, Paris, GF, 2007.

Brasillach, Robert, Corneille, Paris, Fayard, 1938.

Doubrovsky, Serge "Horace ou la conquête de soi", Corneille et la dialectique du héros, Paris, Gallimard, 1963.

Lanson, Gustave, Corneille, Paris, Hachette, 1898.

Herland, Louis, Horace ou la Naissance de l'homme, Paris, Minuit, 1952.

Maurens, Jacques, La tragédie sans tragique. Le néo-stö̈cisme dans l'œuvre de Pierre Corneille, Paris, Armand Colin, 1966.

Sarcey, Francisque, La tragédie, Paris, Libraire des Annales, 1887.

\section{Travaux}

BARONI, Raphaël, «La guerre des voix. Critique polyphonique et divergences interprétatives dans l'œuvre de Michel Houellebecq", Contextes, octobre 2014 (<http://contextes.revues.org/5979>).

-, Les rouages de l'intrigue. Les outils de la narratologie post-classique pour l'analyse des textes littéraires, Genève, Slatkine, 2017.

Bayard, Pierre, Qui a tué Roger Ackroyd?, Paris, Minuit, 1998.

—, Enquête sur Hamlet. Le dialogue de sourds, Paris, Minuit, 2002.

Bionda, Romain, "La vérité du drame. Lire le texte dramatique (Dom Juan)», Poétique, 181 (2017), p. 67-82.

Currie, Gregory, The Nature of Fiction, Cambridge, Cambridge University Press, 1990.

Dolezel, Lubomir, Heterocosmica. Fiction and Possible Worlds, Baltimore/London, The John Hopkins University Press, 1998.

Eco, Umberto, Lector in fabula, trad. M. Bouhazer, Paris, Grasset, 1979. 
Escola, Marc, «Pierre Bayard contre Hercule Poirot, derniers rebondissements dans l'affaire Ackroyd", Acta Fabula, 1/1 (2000) (<http:// www.fabula.org/revue/document7997.php $>$ ).

—, "Le sixième acte. Les seconds amants du théâtre classique», in $L a$ lettre et la scène. Linguistique du texte de théâtre, Dijon, Éditions universitaires de Dijon, 2009, p. 149-158.

—, "Changer le monde», in Françoise Lavocat (éd.), La théorie littéraire des mondes possibles, Paris, CNRS éditions, 2010.

Fish, Stanley, Quand lire c'est faire. L'autorité des communautés interprétatives, Paris, Les Prairies ordinaires, 2007.

Guénoun, Denis, "Cours du 20 décembre 2006 ", in Id., Livraison et délivrance. Théâtre, politique, philosophie, Paris, Belin, 2009, p. 139-148.

Groneberg, Michael, "La mimesis; aspects ludiques et poétiques", in Michael Groneberg (éd,), Penser la scène. Études de Lettres, 306 (2018), p. 145-168.

Herman, David, Storytelling and the Sciences of Mind, Cambridge (Mass.), M.I.T. Press, 2013.

JAHN, Manfred, «Frames, Preferences, and the Reading of Third-Person Narratives; Toward a Cognitive Narratology", Poetics Today, 18/4 (1997), p. 441-468.

Korthal Altes, Liesbeth, Ethos and Narrative Interpretation. The Negotiation of Values in Fictions, Londres, University of Nebraska Press, 2014.

Lewis, David, "Truth in Fiction», American Philosophical Quarterly, 15 (1978), p. 37-46

Maignant, Aurélien, "Juger de la fiction», Poétique, 184 (2018), p. 181194.

NüNnING, Ansgar, "Unreliable Compared to What? Towards a Cognitive Theory of Unreliable Narration", in Walter Grünzweig, Andreas Solbach (dir.), Grenzüberschreitungen: Narratologie im Kontext / Transcending Boundaries: Narratology in Context, Tübingen, Gunter Narr Verlag, p. 53-73.

Pavel, Thomas, Univers de la fiction, Paris, Seuil, «Poétique», 1998.

Russell, Bertrand, "De la dénotation", in Écrits de logique philosophique, Paris, PUF, 1989 (1905). 
Ryan, Marie-Laure, "Cosmologie du récit», in Françoise Lavocat (éd.), La théorie littéraire des mondes possibles, Paris, CNRS éditions, 2010, p. 53-81.

Saint-Gelais, Richard, Fictions transfuges, Paris, Seuil, 2011.

Walton, Kendall, Mimesis as Make-Believe, on the Foundation of

Representational Arts, Londres, Harvard University Press, 1990. 